\title{
Application of pleural flaps in laparoscopic-thoracoscopic esophagectomy for esophageal cancer
}

\author{
Xiaofeng Chen, Shuoyan Liu, Peng Chen, Hao He, Feng Wang \\ Department of Thoracic oncology surgery, Fujian Cancer Hospital \& Fujian Medical University Cancer Hospital, Fuzhou 350014, China \\ Contributions: (I) Conception and design: F Wang; (II) Administrative support: X Chen, S Liu; (III) Provision of study materials or patients: X Chen, \\ S Liu; (IV) Collection and assembly of data: P Chen, H He; (V) Data analysis and interpretation: X Chen; (VI) Manuscript writing: All authors; (VII) \\ Final approval of manuscript: All authors. \\ Correspondence to: Feng Wang, MS. Department of Thoracic oncology surgery, Fujian Cancer Hospital \& Fujian Medical University Cancer Hospital, \\ Fuzhou 350014, China. Email: wfmd120@163.com.
}

\begin{abstract}
Background: This study aimed to investigate the clinical efficacy of pleural flaps usage in laparoscopicthoracoscopic esophagectomy for esophageal cancer.

Methods: Six hundred and nineteen patients received esophagectomy for esophageal cancer. All these 619 patients received laparoscopic-thoracoscopic esophagectomy. These 304 patients (study group) used pleural flaps and the other 315 patients (control group) had no pleural flaps. The observation indicators were postoperative complications, including cervical subcutaneous emphysema, fistula of cervical anastomosis, and anastomotic leakage into the pleural cavity.

Results: In the study group, 5 patients had cervical subcutaneous emphysema after surgery $(1.64 \%)$ compared to 38 patients in the control group $(12.06 \%)$, which showed significant difference $(\mathrm{P}<0.05)$. Moreover, the study group displayed 1 case of anastomotic leakage into the pleural cavity $(0.33 \%)$, compared with 8 patients in the control group $(2.54 \%)$, which showed significant difference $(\mathrm{P}<0.05)$. Logistic regression indicated that the application of pleural flaps effectively reduced the incidence of postoperative complications.
\end{abstract}

Conclusions: Covering the upper mediastinum with pleural flaps effectively reduced the incidence of cervical subcutaneous emphysema and anastomotic leakage into the pleural cavity.

Keywords: Pleural flaps; cervical subcutaneous emphysema; fistula of cervical anastomosis; anastomotic leakage into the pleural cavity

Submitted May 05, 2019. Accepted for publication Nov 19, 2019.

doi: $10.21037 /$ jtd.2019.12.65

View this article at: http://dx.doi.org/10.21037/jtd.2019.12.65

\section{Introduction}

The new patients and deaths of esophageal cancer in China accounted for over half of the total new patients and deaths in the world, and surgery remains to be the first treatment choice $(1,2)$. With the popularization of thoracoscopy and laparoscopy, the combination of laparoscopic-thoracoscopic esophagectomy for the treatment of esophageal cancer has achieved good efficacy and good surgical outcome, and has been widely used. During the surgical process, anastomosis of the digestive tract is considered as an important step (3).
In the present study, the incidence of anastomosisrelated complications, such as symptomatic anastomosis leakage, anastomosis bleeding, anastomotic fistula, was reported to be above $10 \%$, implying high risk of severe cervical anastomotic leakage into the pleural cavity (4). To investigate the clinical efficacy of the pleural flaps during esophageal cancer surgery, we collected the clinical data from 619 patients, who receive laparoscopic-thoracoscopic esophagectomy in our department from January 2012 to January 2018. In this present study, we will retrospectively review and analyze the whole data, which aim to provide 
the guidance in surgical process and improve the patents' welfare and surgical outcome.

\section{Methods}

\section{Operative technique}

In the three-field operation for esophageal cancer, we firstly dissociated the esophagus through the right chest and then dissected the regional lymph nodes especially the left and right recurrent para laryngeal lymph nodes. Subsequentially, the stomach was supine and dissociated, and then made into a tubular stomach. The thoracic cavity and abdominal cavity were completed by endoscopy. In the perioperative process, a neck anastomotic fistula may be shaped at the at the anastomosis of neck esophagus and stomach. If the anastomotic fistula is confined to the neck, the patient's systemic infection symptoms were mild. If the anastomotic fistula leaked into the chest, the patient's systemic infection symptoms were severe and the mortality rate was high. Patients often have cavities in the cervical-thoracic junction. The incidence of intrathoracic leakage and subcutaneous emphysema in the neck anastomotic approach was significantly increased due to the application of fine tube stomach and the dissection of bilateral recurrent laryngeal nerve lymph nodes. When the chest pleural triangular flap jacking is free in the surgery on mediastinal, we would open on the right side of front and azygos vein arch on the edge of the vagus nerve pleura, keep the lateral wall on the front of the pleura, line into the chest pleural triangular flap. After the surgery, we interrupted the suture pleural, covered on the later of the tubular stomach, gastric tube and promoted the lift wall layer pleural adhesion as soon as possible, closed chest and cleared the neck traffic forms.

\section{Baseline data}

A retrospective case-control study was conducted by collecting the clinical data of 619 patients with stage T1-3 esophageal cancer who received surgical treatment at the Department of Thoracic Surgery of Fujian Provincial Cancer Hospital from January 2012 to January 2018. Among them, 488 were males and 131 were females, and were aged $57.45 \pm 7.08$ years old. All of them were preoperatively confirmed as having esophageal squamous cell carcinoma by gastroscopy. Of the 619 patients, 95 had lesions in the upper segment, 411 had lesions in the middle segment, and 113 in the lower segment. Randomized concurrent control study was performed for retrospective analysis. All 619 patients received mechanical anastomosis (side-to-end anastomosis between the esophagus and posterior wall of the stomach, with Johnson \& Johnson circular stapler). According to the inclusion and exclusion criteria, pleural flaps were used in 304 patients (study group, 240 males and 64 females, aged $18-75$ years, with an average of $55.56 \pm 7.92$ years), and were not used in 315 patients (control group, 248 males and 67 females, aged 20-70 years, with an average of $53.78 \pm 8.64$ years). The present study was approved by the ethics committee, and the informed consent was signed by all patients before undergoing surgery.

\section{Inclusion and exclusion criteria}

Inclusion criteria: patients (I) who were pathologically diagnosed as esophageal cancer by preoperative gastroscopy; (II) who received preoperative barium meal examination, contrast-enhanced CT scan of the chest and abdomen, ECT, chest X-ray and B-mode ultrasonography of the abdomen. The external invasion and distant metastasis of the tumor, and cervical, mediastinal and celiac lymph node enlargement were assessed. The preoperative staging was T03N02M01; (III) who received preoperative ECG and lung function test and assessment of anesthetic and surgical tolerance; (IV) who underwent subtotal resection of the esophagus via the right chest incision + gastric dissociation in the upper abdomen + esophagogastric anastomosis, with cervical, thoracic and abdominal lymph node dissection; and who achieved R0 resection as indicated by postoperative pathology (i.e., there were no residual tumor cells by gross observation or under the microscope). Patients with the following conditions were excluded: (I) who received preoperative neoadjuvant radiochemotherapy; (II) had severe complications of heart, lungs, liver or kidneys; (III) who have received palliative resection; (IV) who received lymph node dissection, including systematic mediastinal and abdominal lymph nodes; (V) who had stage T4a by postoperative pathology. TNM staging was performed according to the eighth version of AJCC/UICC TNM staging system.

\section{Surgery and anastomosis}

Surgical methods: Gastrointestinal decompression tube was inserted before surgery and general anesthesia was performed. All staplers used in the surgery were purchased from Johnson \& Johnson, and all the surgeries were performed by the same group of thoracic surgeons. The 

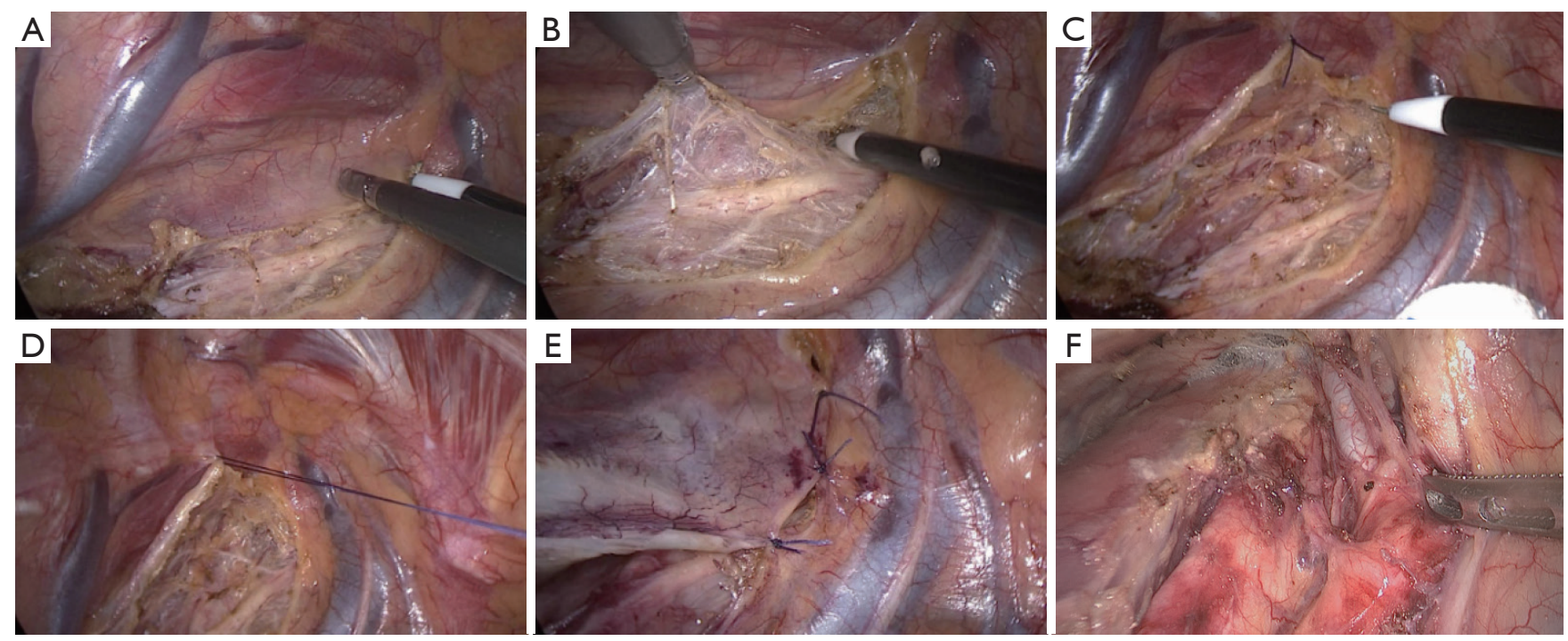

Figure 1 The operation of pleural flaps.

details of laparoscopic-thoracoscopic esophagectomy were described in the previous literature (2). Pleural flaps were harvested in the pleural cavity, i.e., the upper mediastinal pleura was opened in front of the right vagus nerve and on the superior margin of the arch of azygos vein within the pleural cavity. Specifically, the parietal pleura was not opened on the lateral margin of the spine, and only the pleura in front of the right vagus nerve and on the superior margin of the arch of azygos vein was opened to form a pleural flap (Figure 1A,B). The flap could be immobilized to the lateral chest wall with sutures during surgery to avoid disturbance to the mediastinal exposure and lymph node dissection (Figure 1C,D). When the thoracic surgery was completed, the pleura was sutured intermittently to restore its original state (Figure 1E). The flap was then covered on the tubular stomach, which was then pulled up to form the pleural flap. Jejunostomy was performed and enteral nutrition was started at $24 \mathrm{~h}$ after surgery.

Main equipment such as stapler (Johnson \& Johnson, Beijing Fangdahezhong Technology Development Co., Ltd., China), model ec60a/ec45a; thoracoscope (Leica, Wuhan Tianzeweiye Medical Equipment Co., Ltd., China), model SY-SHREK, medicine (device) license: Hubei 2015 (No. 0286); laparoscope (Nanjing Sufutong Technology Co., Ltd., China), model LNS230/LNS231/L.

Mechanical anastomosis: End-to-side anastomosis was performed between the esophagus and posterior wall of stomach (Johnson \& Johnson, circular stapler, 25\#). The stub was closed and embedded with a closer (Changchou, disposable).

\section{Diagnostic criteria}

Anastomotic leakage was highly suspected if fever along with redness, swelling and exudation from the cervical incision and with drainage of turbid liquid. The diagnosis was made and the fistula was identified based on the gastrointestinal tract radiography, CT scan and gastroscopy. Thoracic-gastric fistula was excluded. Anastomotic leakage into the mediastinum was defined as communication between the anastomotic fistula and mediastinum, which was only confined to the mediastinum, but not communicated with the pleural cavity. Anastomotic leakage into the pleural cavity was defined as the communication between the anastomotic fistula and pleural cavity, and with the drainage of pus or digestive juice via the chest tube and systematic infection, surgery or puncture and drainage.

\section{Observation indicators}

The number and percentage of patients with cervical subcutaneous emphysema, fistula of cervical anastomosis, and anastomotic leakage into the pleural cavity were calculated.

\section{Statistical analysis}

Statistical analysis was performed using SPSS 20.0 software. The rates were compared by four-fold table $\chi^{2}$ test or Fisher's exact test. Quantitative data were analyzed by $t$ 
Table 1 Comparison of clinicopathological data between the two groups

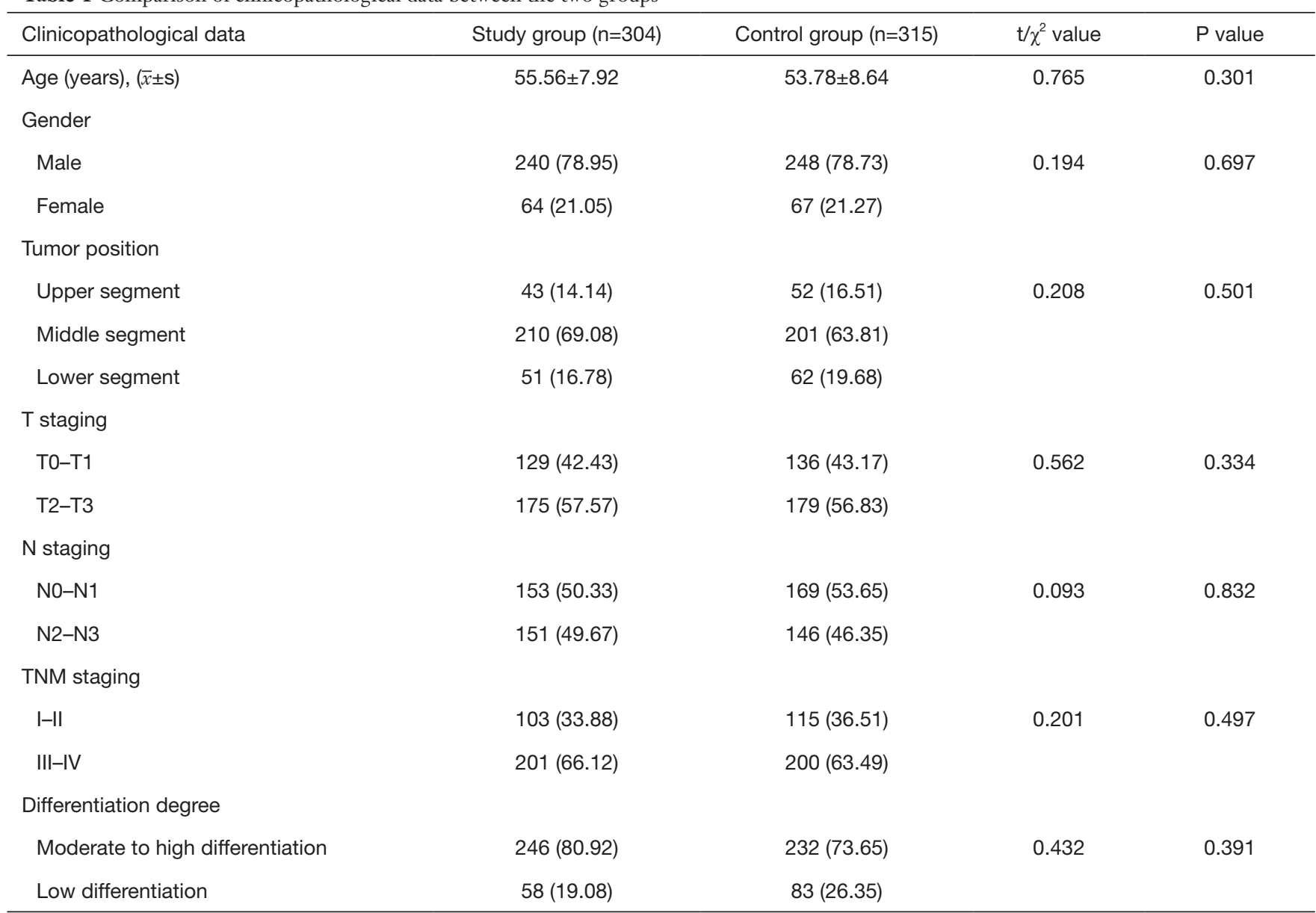

test and expressed as $\bar{x} \pm \mathrm{SD} . \mathrm{P}<0.05$ indicated statistically significant differences.

\section{Results}

\section{Comparison of baseline information between the two groups}

There was no significant difference in age, gender, tumor position, differentiation degree, $\mathrm{T}$ staging, $\mathrm{N}$ staging and TNM staging between the two groups $(\mathrm{P}>0.05$, Table 1$)$.

\section{Comparison of postoperative complications between the two groups}

The study group had 5 patients with cervical subcutaneous emphysema (1.64\%) when compared to 38 patients $(12.06 \%)$ in the control group, and showed significant differences $(\mathrm{P}<0.05)$. There were 12 patients $(3.95 \%)$ with fistula of cervical anastomosis in the study group compared to 14 patients $(4.44 \%)$ in the control group, and showed no significant difference $(\mathrm{P}>0.05)$. There was 1 case $(0.33 \%)$ of anastomotic leakage into the pleural cavity in the study group compared to 8 patients $(2.54 \%)$ in the control group, showing significant difference $(\mathrm{P}<0.05)$ (Table 2).

\section{Correlation between complications and surgical approach}

Whether the occurrence of postoperative complications was taken as the dependent variable $(0=$ no, $1=$ yes $)$, and covariates including gender, age, TNM staging, tumor position, $\mathrm{T}$ staging, $\mathrm{N}$ staging and differentiation degree were controlled. The factor of whether the pleural flaps were used or not in the laparoscopic-thoracoscopic esophagectomy was included into the unconditional multivariate logistic regression model. Compared with the "yes" group, the variables of the logistic model in the "no" 
Table 2 Comparison of postoperative complications between the two groups

\begin{tabular}{|c|c|c|c|c|}
\hline Type of complications & Study group $(n=304)$ & Control group $(n=315)$ & $\chi^{2}$ & $P$ \\
\hline Fistula of cervical anastomosis & 12 & 14 & 0.10 & $>0.05$ \\
\hline Anastomotic leakage into the pleural cavity & 1 & 8 & 0.04 & $<0.05^{*}$ \\
\hline
\end{tabular}

*, using Fisher's exact test.

group were shown as followings: $\beta-1.203$; OR $0.331 ; 95 \%$ CI, 0.478-0.944; $\mathrm{P}$ value $<0.001$. The results showed that the use of pleural flaps in the laparoscopic-thoracoscopic esophagectomy was a protective factor for postoperative complications.

\section{Discussion}

Along with the advances and popularization of hysteroscopy, the laparoscopic-thoracoscopic esophagectomy has achieved good efficacy. Multi-center, randomized control studies have shown that the minimally invasive surgery for esophageal cancer outperforms the conventional open surgery in terms of the incidence of pulmonary complications (5-7). Moreover, the patients' quality of life at 1 year after surgery was also superior to that of conventional open surgery during the follow-up period (6). Digestive tract anastomosis during surgery is regarded as an important step in the reconstruction of digestive tract. It has been reported that the incidence of cervical anastomosis-related complications was over $10 \%(8,9)$. Fistula of cervical anastomosis mainly presented local infection, subcutaneous emphysema and abscess. When lying superficially, the fistula can be more likely discovered timely and drained. The cervical blood supply restores after surgery, the infection disappears and the anastomotic fistula remains easy to be healed (10). In clinic, cervical anastomotic leakage into the mediastinum and pleural cavity requires special attention, especially for severe complications such as leakage into the pleural cavity. Infections caused by complications further cause chest pain, breathing difficulty, high fever, rapid pulse and septic shock, prolonging the hospital stay and costs, aggravating the patient's conditions and increasing mortality (11). It has been shown that the mortality related to complications varied between $11 \%$ and $35.7 \%(12,13)$.

Fistula is the prerequisite for leakage, and hence it is necessary to reduce the anastomotic fistula. However, in the laparoscopic-thoracoscopic esophagectomy for esophageal cancer, the reasons for postoperative fistula of the cervical anastomosis are very complex. Surgical time, anastomotic methods and pathway, preoperative nutritional status, underlying diseases, preoperative neoadjuvant radiotherapy and postoperative nutritional support may play a role (14). Along with the progression of surgical skills and experience, the relevant complications can be reduced. But whatever the anastomotic technique is used, it is still impossible to completely avoid anastomotic fistula. Based on the long-term clinical experience of our center, whether for manual anastomosis or mechanical anastomosis, the probability of fistula usage of cervical anastomosis is controlled by about $3 \%$ and $5.8 \%$, respectively (15). In recent years, tubular stomach, especially thin tubular stomach has gradually replaced the whole or almost the whole stomach in esophagogastric anastomosis. The former has achieved a satisfactory effect in reducing or avoiding leakage into the pleural cavity and thoracic-gastro syndrome (16-18). But after the laparoscopic-thoracoscopic esophagectomy for esophageal cancer, anastomotic leakage into the mediastinum and pleural cavity seemed to occur more frequently. This is probably due to the residual cavity formed by lymph node dissection along the right recurrent laryngeal nerve in the apex (Figure $1 F$ ) and the use of thin tubular stomach. Conventionally, the upper and posterior mediastinal pleura are usually resected along with the esophagus. However, the local cavity formed by the resection communicates with the cervicothoracic junction. The air likely spreads to the neck via the pleural cavity during the early stage after surgery. If anastomotic fistula occurs at the later stage, the resulting infection is less likely to be confined, but spreads to the mediastinum and the pleural cavity. To prevent this, the pleural flaps were prepared during surgery and were immobilized to the lateral chest wall using sutures, avoiding disturbance to the subsequent exposure of upper and posterior mediastinum and lymph node dissection. The pleura was sutured intermittently after surgery to restore the original state. The flap was covered on the tubular stomach, which was then pulled up. This method can promote adhesion between 
the pulled-up tubular stomach and parietal pleura, closing the gap formed by communication between apex and the neck. This in turn reduces the early incidence of cervical subcutaneous emphysema. Singla (19) reported that after the mediastinum becomes a part of the pleura, the tubular stomach can be well immobilized into the mediastinum, reducing the tension of tubular stomach. As a result, the tubular stomach does not move and can be subjected to traction with gravity, swallowing and peristalsis. A better protection can be offered to the anastomosis as the tension of the anastomosis is reduced, which is conducive to the healing of anastomotic fistula. The parietal pleural deltoid flap itself cannot reduce the incidence of neck anastomotic fistula. Even if anastomotic fistula occurs, the infection can be confined to the neck and systemic poisoning symptoms can be reduced by pleural flaps. Besides, logistic regression indicated that the use of pleural flaps to cover the upper mediastinum in the laparoscopic-thoracoscopic esophagectomy for esophageal cancer effectively reduced the postoperative complications. This was probably due to the restoration of the upper mediastinum to the original state with the use of flaps by eliminating lacuna, and this remains conducive to early atresia of pleural cavity. For this reason, the pleural flaps are used to cover the upper mediastinum and can reduce the incidence of anastomotic leakage into the mediastinum, thus avoiding thoracic cavity leakage and the adverse systemic impact of the complications. Moreover, this procedure is easy to perform and does not prolong surgical time or add to the difficulty of lymph node dissection or the overall risk of the surgery. Based on the hints from Gottlieb-Vedi et al., the longterm survival after MIE may be improved by pleural flaps preservation (20). Besides, it is possible that patients can have better global quality of life, physical function, fatigue and pain after minimally invasive surgery $(20,21)$.

Although there are different advantages by using this method, there are some limitations. The preservation of pleural flaps was only applied to stage T0-3 patients. The patients were already present in stage $\mathrm{T} 4 \mathrm{a}$ if the tumor had affected the mediastinal pleura, and the affected pleural must be completely resected $(22,23)$. Moreover, while dissociating the pleural flaps, the surrounding adipose tissues must be completely resected as well to avoid residual lymphatic and adipose tissues. The pleural flaps must be kept intact in order to have a sealing effect. In addition, the operation cannot be performed if no enough pleura is retained in some patients with esophageal cancer invasion into the upper mediastinal pleura. Meanwhile, the parietal pleural deltoid flap itself can't reduce the incidence of neck anastomotic fistula.

To conclude, the application of pleural flaps to cover the upper mediastinum may reduce the risk of anastomotic leak to pleural cavity and emphysema after laparoscopicthoracoscopic esophagectomy for esophageal cancer. It is extremely possible that this method could effectively prevent postoperative cervical subcutaneous emphysema and cervical anastomotic leakage into the pleural cavity without disrupting the progression of surgery. The analysis of the application of this method would hopefully contribute to its clinical popularization.

\section{Acknowledgments}

Funding: The study was supported by the Wu Jieping Medical Foundation-Clinical Scientific Research Project, China, No. 320.2730.1881; Medical Innovative Project of Fujian Province, China, No2018-CXB-3.

\section{Footnote}

Conflicts of Interest: The authors have no conflicts of interest to declare.

Ethical Statement: The authors are accountable for all aspects of the work in ensuring that questions related to the accuracy or integrity of any part of the work are appropriately investigated and resolved. The present study was approved by the Fujian Provincial Cancer Hospital Ethics Committee (YKT2019-014-01), and the informed consent was signed by all patients before undergoing surgery.

Open Access Statement: This is an Open Access article distributed in accordance with the Creative Commons Attribution-NonCommercial-NoDerivs 4.0 International License (CC BY-NC-ND 4.0), which permits the noncommercial replication and distribution of the article with the strict proviso that no changes or edits are made and the original work is properly cited (including links to both the formal publication through the relevant DOI and the license). See: https://creativecommons.org/licenses/by-nc-nd/4.0/.

\section{References}

1. Research report on risk factor of cancer in China. Beijing: 2003. 
2. Liu SY, Wang F, Zheng QF, et al. Clinical application of minimally invasive esophagectomy for esophageal carcinoma. Zhonghua Wei Chang Wai Ke Za Zhi 2012;15:947-9.

3. Xu M, Liu C, Guo M, et al. Combined laparoscopicthoracoscopic esophagectomy and intrathoracic esophagogastric anastomsis. J Thorac Dis 2013;5:895-7.

4. Kutlu CA, Olgac G. Pleural flap to prevent lobar torsion: A novel technique. Eur J Cardiothorac Surg 2006;30:943-4.

5. Biere SS, van Berge Henegouwen MI, Maas KW, et al. Minimally invasive versus open oesophagectomy for patients with oesophageal cancer: a multicentre, open-label, randomised controlled trial. Lancet 2012;379:1887-92

6. Simonin A, Gonzalez M, Levivier M, et al. Letter to the editor: dural reconstruction with a vascularised pleural flap following en bloc resection of a thoracic epithelioid sarcoma: technical note. Acta Neurochir (Wien) 2014;156:1223-4.

7. Fricke A, Bannasch H, Klein HF, et al. Pedicled and free flaps for intrathoracic fistula management. Eur J Cardiothorac Surg 2017;52:1211-7.

8. Maas KW, Cuesta MA, van Berge Henegouwen MI, et al. Quality of Life and Late Complications After Minimally Invasive Compared to Open Esophagectomy: Results of a Randomized Trial. World J Surg 2015;39:1986-93.

9. Sun HB, Li Y, Liu XB, et al. Early Oral Feeding Following McKeown Minimally Invasive Esophagectomy: An Openlabel, Randomized, Controlled, Noninferiority Trial. Ann Surg 2018;267:435-42.

10. Kirshenbaum EJ, Zhao LC, Myers JB, et al. Patency and Incontinence Rates After Robotic Bladder Neck Reconstruction for Vesicourethral Anastomotic Stenosis and Recalcitrant Bladder Neck Contractures: The Trauma and Urologic Reconstructive Network of Surgeons Experience. Urology 2018;118:227-33.

11. Huang J, Zhou Y, Wang C, et al. Logistic regression analysis of the risk factors of anastomotic fistula after radical resection of esophageal-cardiac cancer. Thorac Cancer 2017;8:666-71.

12. Wang J, Zhang M, Pan W, et al. Management of recurrent tracheoesophageal fistula after esophageal atresia and follow-up. Dis Esophagus 2017;30:1-8.

13. Junemann-Ramirez M, Awan MY, Khan ZM, et al. Anastomotic leakage post-esophagogastrectomy for esophageal carcinoma: retrospective analysis of predictive factors, management and influence on longterm survival in a high volume centre. Eur J
Cardiothorac Surg 2005;27:3-7.

14. Lange B, Sold M, Kähler G, Wessel LM, Kubiak R. Experience with fully covered self-expandable metal stents for anastomotic stricture following esophageal atresia repair. Dis Esophagus 2018. doi: 10.1093/dote/doy061.

15. Wang F, Liu S, Wang J, et al. Comparison of the stapled suture with the manual suture in the application of minimally invasive esophagectomy. Chin J Gastrointest Surg 2014;(9):881-3.

16. He J, Chen M, Shao W, et al. Surgical Management Of 3 Cases With Huge Tracheoesophageal Fistula With Esophagus Segment in situ As Replacement Of The Posterior Membranous Wall Of The Trachea. J Thorac Dis 2009;1:39-45.

17. Saitoh T, Takamura A, Watanabe G. Endoscopic and clinicopathological features of intramucosal, histologically mixed-type, low-grade, well-differentiated gastric tubular adenocarcinoma with the potential for late-onset lymph node metastasis. BMC Gastroenterol 2018;18:189.

18. Mitsuishi T, Hamatani S, Hirooka S, et al. Clinicopathological characteristics of duodenal epithelial neoplasms: Focus on tumors with a gastric mucin phenotype (pyloric gland-type tumors). PLoS One 2017;12:e0174985.

19. Singla N, Kaman L, Singh R. Anterior route reconstruction after transhiatal esophagectomy is comparable with posterior route reconstruction. Indian J Gastroenterol 2007;26:143-5.

20. Gottlieb-Vedi E, Kauppila JH, Malietzis G, et al. Longterm Survival in Esophageal Cancer After Minimally Invasive Compared to Open Esophagectomy: A Systematic Review and Meta-analysis. Ann Surg 2019;270:1005-17.

21. Kauppila JH, Xie S, Johar A, et al. Meta-analysis of healthrelated quality of life after minimally invasive versus open oesophagectomy for oesophageal cancer. Br J Surg 2017;104:1131-40.

22. Adachi H, Ueda N, Mizukami Y, et al. [Surgical Treatment of T3 Lung Cancer with Invasion of Chest Wall and Other Adjacent Organs]. Kyobu Geka 2017;70:898-902.

23. Tabatabaei SA, Hashemi SM, Kelidari B. Transhiatal Esophagectomy without Mediastinal Manipulation for Lower Third Esophageal and Cardial Cancers: The First Experience of a New Technique. Iran J Cancer Prev 2015;8:89-93.

Cite this article as: Chen $\mathrm{X}$, Liu S, Chen P, He H, Wang F. Application of pleural flaps in laparoscopic-thoracoscopic esophagectomy for esophageal cancer. J Thorac Dis 2020;12(3):973-979. doi: 10.21037/jtd.2019.12.65 